\title{
sciendo
}

\section{ANOMALY OF ROTOR DYNAMICS IN ULTRA-LIGHT HELICOPTER - ROBINSON R22}

\author{
Agnieszka Sobieszek \\ Lukasiewicz Research Network - Institute of Aviation \\ Department of Transport and Energy Conversion Division \\ Krakowska Av. 110/114, 02-256 Warsaw, Poland \\ tel.: +48228460011 , ext. 224, fax: +48228464432 \\ e-mail: agnieszka.sobieszek@ilot.edu.pl
}

\begin{abstract}
The article presents the analysis of anomaly of rotor dynamics in ultra-light helicopter - Robinson R22. Robinson $R 22$ is two-seat, two-blade main rotor and single-engine helicopter, well known as simple and common used aircraft because of low price and high availability. At the same time, large number of accidents and strictly defined rules (recommended piloting technique) and weather condition for safe flight show disadvantages of Robinson R22. The reason for considering this topic is the analysis of different flight properties and helicopter behaviour as well as easy entering into dangerous flight manoeuvre. In the article different flight properties and loss of control during the pull-up, manoeuvre or vertical gusts of wind and mast bumping accidents were analysed. Analysis shows that problem may be caused by construction of three-hinged rotor hub, designed and patented by F. Robinson. Article presents model of rotor hub and review of main rotor and rotor hub construction in light helicopters. Because of number of accidents, caused by the unusual behaviour of Robinson R22, restrictive pilotage rules were introduced: prohibition of flight in certain weather conditions, the necessity of attending additional training conducted by trained instructors. To reduce the probability of an accident a special instruction for specific Robinson R22 properties was created. Moreover, the statistics of accidents resulting from loss of control and review of legal changes caused by Robinson $R 22$ accidents are presented.
\end{abstract}

Keywords: rotor hub, Robinson R22, helicopter, the pull-up manoeuvre, rotor dynamics

\section{Introduction}

Basing on the main rotor's dynamics analysis, this article aims to specify the reasons of high accident ratio, which was observed for Robinson R22. This helicopter is defined as difficult, taking into account a serious number of accidents caused by different pilot characteristics and helicopter behaviour during the flight. In opposite the other ultralight helicopters, R22 much often gets into dangerous flight condition. The explanation for this problem can be linked with in unusual construction of teetering, semi-rigid system of rotor hub.

The study identifies what caused Robinson R22 becoming more vulnerable to accidents over other ultralight helicopters. The results were achieved by analysing reports from the air accidents and research within experienced pilots. Reported air incidents were group into two buckets - LOC (Loss of control in flight) and non-LOC. Statistically, the number of non-LOC accidents for Robinson R22 is comparable to the number of accidents of similar type of helicopters (i.e. Bell 47 as the construction and flight parameters are close to R22). On the other side, comparing category of LOC accidents a significant variation was observed. There were almost six times more Robinson R22 incidents in reference to the other models. Considering the given information, Robinson R22 is the most dangerous helicopter with the highest accident ratio per 100,000 flight hours with the result 1,509 (before introducing changes mentioned below ratio was 6 - in 1982), whereas this coefficient is much lower for the helicopter of this type: 0,015 for Bell 206, 0,201 Bell 212, 0,439 - Bell 204) [3, 4].

Because of high accident ratio in the first years of use, Robinson Company introduced safety 
program and a number of changes [6]:

- the number of flight hours for instructors has been increased (to 150-500 h),

- Safety Notices was prepared and sent to all R22 owners,

- added $0.72 \mathrm{~kg}$ to the tips of main rotor blades to increase internal energy,

- in 1990 started offering a governor to help eliminate low rotor rpm, and in 1995 started installing governor as a standard equipment,

- FAA passed SFAR 73 - stricter time and instruction standards for anyone flying a Robinson R22 helicopter.

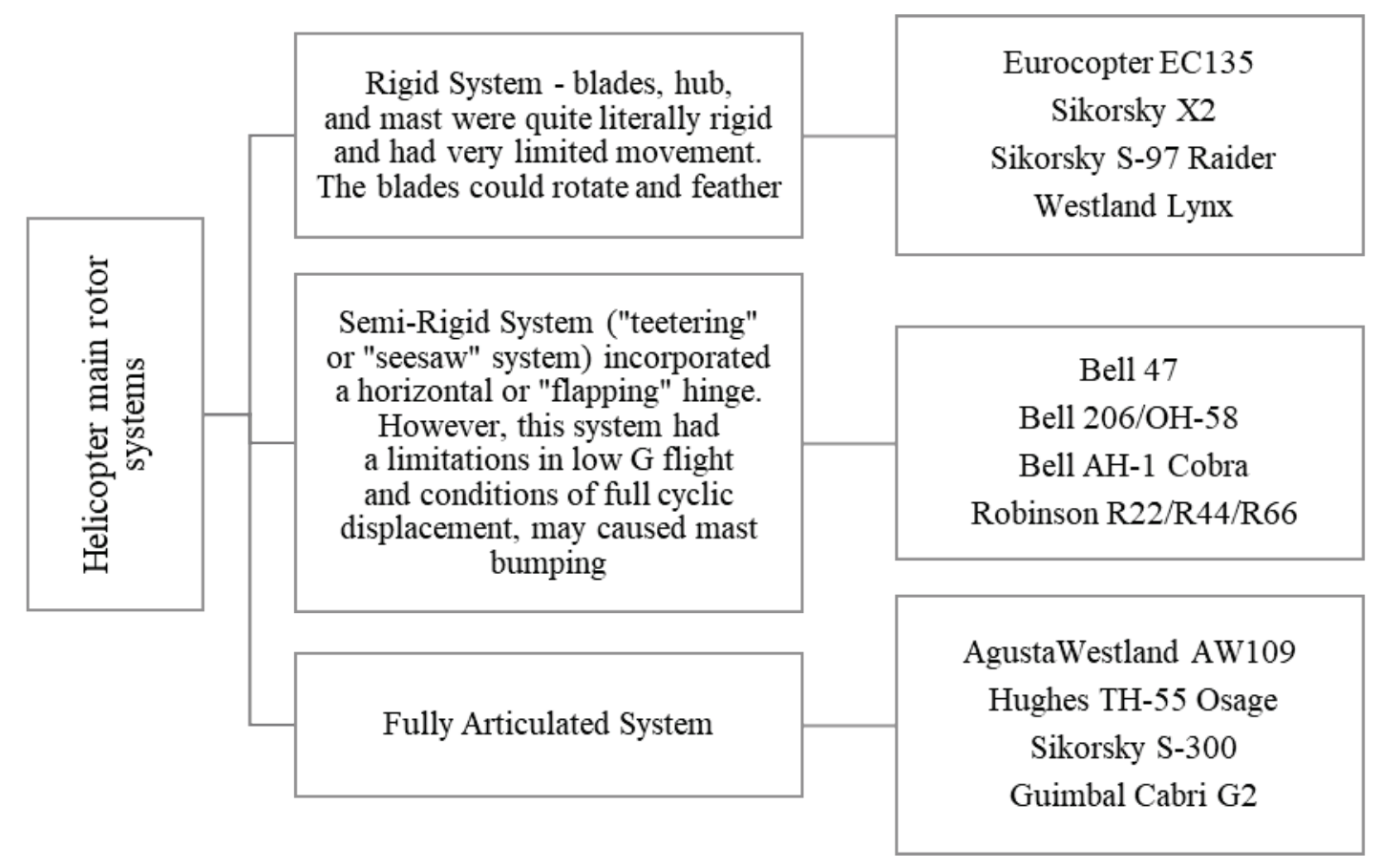

Fig. 1. System of helicopter main rotor, definitions and examples [1, 7]

\section{Causes of Robinson R22 accidents}

Analysing a significant number of Robinson's R22 accidents for main reasons (caused by loss of control in flight) the following has been identified: loss of main rotor control, structural failure of the rotor blade, fatigue of rotor blade materials, accidents related to meteorological conditions (mainly windy weather), basic construction of the rotor hub which can lead to mechanical defects, excessive discrepancy between the position of the rotor blades, mast bumping or oversensitive control system.

Considering the differences between Robinson R22 and other ultralight, two-rotor blade helicopters it is worth to focus on rotor hub construction (Fig. 2). Robinson R22 rotor hub is light, semi-rigid (teetering) system. For comparison, in Bell UH-1 and Bell 206, the bending motion on the one of the rotor blades automatically causes the upward movement of the second blade and vice versa, R22 rotor hub design enables independent bending movements on both blades. Accidents caused by divergence of the rotor blades constitute a large percentage of Robinson R22 accidents.

Despite the facts, it was found that those are not the mechanical causes, which affect this type of accident, but the human factor. Claim that such a rotor blade work is typical for R22 helicopter.

Robinson R22 is characterized by high control sensitivity, coupling between individual control channels and dynamic motors, that is why flight instructor focus on state of the rotor low speed and ability to exit them during pilots training. If the instructor holds (in mentioned situation) the control rod and is able to prevent large, sudden manoeuvres, a quick response can lead the helicopter out of an unusual condition and prevent an accident. However, sometimes proper main 
rotor low speed and unusual behaviour of the rotor are hard to recognize even for qualified pilot, then lack of fast response caused excessive discrepancy between the position of the blades. Main difference between R22 and other ultralight helicopters is a much higher required rotor speed and the relatively lower rotor blade kinetic energy due to their weight. Low speed of main rotor to certain extent may cause dangerous state of flight and as a result mast bumping, in such case, in Robinson R22 rotor blade may collides with the fuselage or tail boom.

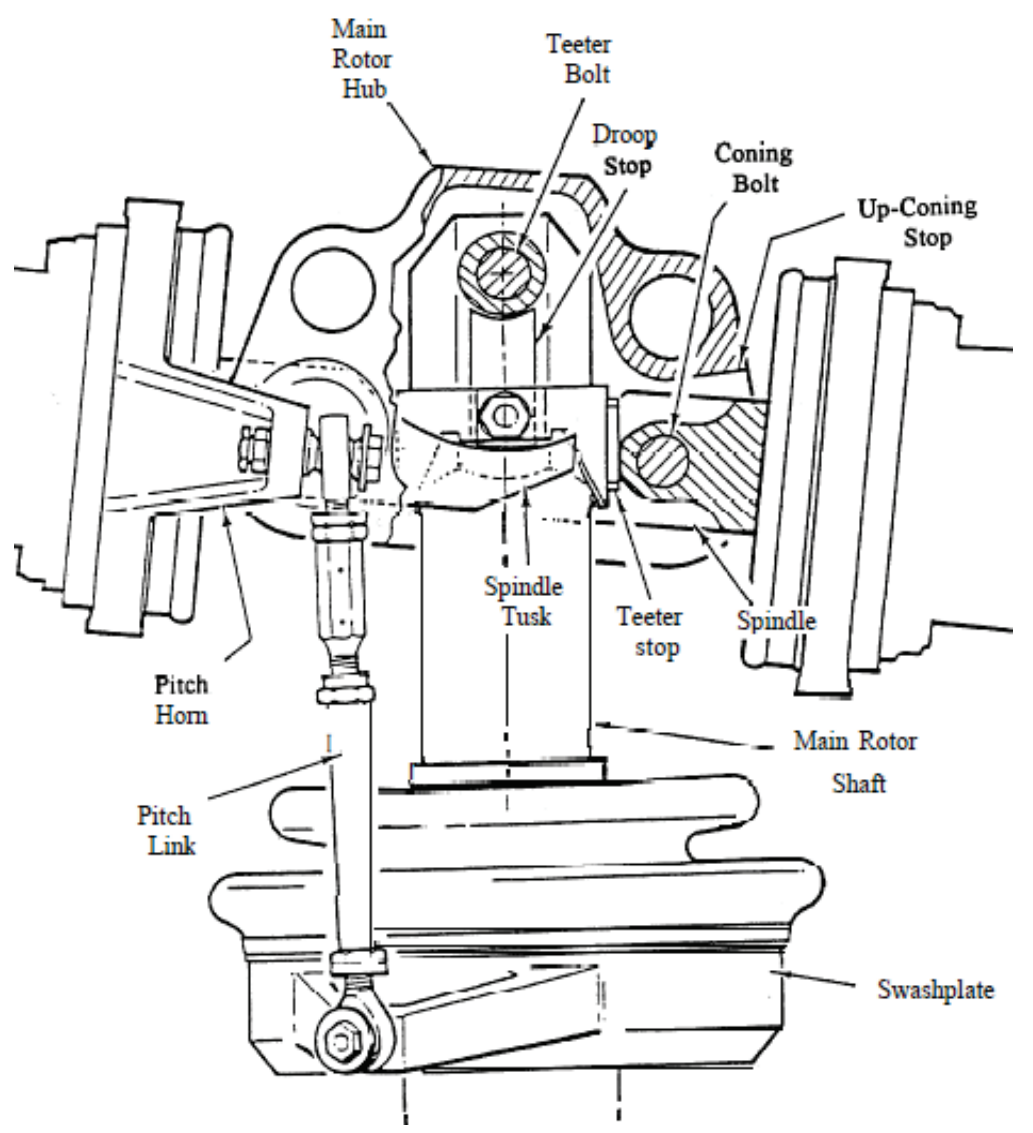

Fig. 2. Scheme of the Robinson R22 rotor hub [3]

Problems with helicopter control can occur when the helicopters Robinson R22 is overloaded (621 kg in this case). With such a mass, the angle of attack reaches almost a critical value. If sudden controlling occurs during overload flight angle of attack reaches critical value and the rotor speed will decrease immediately.

Robinson R22 has semi-rigid (teetering) system, with a range of 12 degrees. Most bumping, just as the name implies is where the main rotor hub contacts or bumps the rotor mast. This causes damage to the mast (in extreme cases can cause catastrophic damage resulting complete rotor separation). Low-G flight is one of the reasons of mast bumping and may occur during pull-up manoeuvre. What is important, fully articulated and rigid rotor systems can do this type of manoeuvre, teetering rotor systems cannot without risking mast bumping. Process of mast bumping in semi-rigid looks like the following:

- when Robinson R22 reaches highest point during manoeuvre, reaches weightlessness known as Low-G or zero-G and rotor speed decrease immediately,

- thrust of tail rotor does not change value, so main rotor thrust cannot counteract and helicopter side-slips rolls towards the right,

- even when the pilot recognizes this situation and tries to apply more left cyclic, it may not be possible because main rotor is unloaded and resulting crossing the limiters and rotor hub and mast contact (mast might be bend or break). 
The best solution to prevent mast bumping in Robinson R22 is to avoid pushover-type manoeuvres. If the use of such a manoeuvre is unavoidable and pilot can recognize Low-G, should immediately and smoothly apply aft cyclic to load the rotor and establish level flight. Untypical helicopter behaviour is not defined as dangerous but as requiring specific skills. Robinson Company claims that state of Low-G is acceptable when the R22 is piloting in a reasonable way and ensuring the dynamic stability of the helicopter. A critical situation, such as a mast stroke, can only occur under aggressive control conditions.

R22 helicopter control requires great precision due to the ease of stalling rotor. The high reactivity and sensitivity of $\mathrm{R} 22$ for cyclic and violent controlling can lead to the mast bumping and rotor blades strike the fuselage. It is possible that even a temporary redirection of attention for experienced pilot (such radio settings) will result in problems with loss of control.

It is worth analysing the impact of weather conditions for which regulations have been tightened, i.e. it is prohibited to fly a Robinson R22 helicopter in windy weather when the wind speed exceeds $37 \mathrm{~km} / \mathrm{h}$. Special procedures are also recommended when flying in windy weather.

\section{Legal regulations of Robinson R22 flights}

After considering all the above-mentioned causes of accidents, defined basis in reports of LOC type helicopter R22 accidents, numerous changes in the field of piloting and training on this type of helicopters were introduced. Over 60 documents with tips and advice to prevent accidents were created. Manuals and training guides for students and instructors were changed aiming to reduce number of accidents.

Despite the obvious problem in Robinson R22 caused by rotor hub construction National Transportation Safety Board (NTSB), which had been dealing with the analysis of accidents, excluded design errors by explaining them with specific pilot properties [4]. Ultimately, no construction errors were found, and NTSB made decision that changes in the training guides for Robinson R22 operators would be sufficient, also, the conditions in which the helicopter could work were limited.

In March 1995 Federal Aviation Administration established a specific training requirement for the R22 (and R44) - Special Federal Aviation Regulation 73 (FAR Part 61 SFAR 73). This tutorial includes explaining the regulation, and training videos discussing energy management, low rotor RPM and rotor stall, and low G mast bumping. SFAR 73 were created and published after NTSB (in January 1995) suspended all flight operations (because of high accident rate) until further research, testing, and modifications were made. Requirements for training and usage included in SFAR 73 contributed to significant decreasing of accidents involving low RPM or low G from previous years. In March 2008 SFAR 73 provided a permanent addition to Part 61 [8].

\section{Simplified rotor hub model}

In order to evaluate the rotor hub's work, simplified model and existing destabilizing forces were analysed (Fig. 3). The forces affecting destabilization are F1 - rotor blades lift force and the centrifugal force of the hub F2 while the stabilizing forces are the centrifugal force of the blades F3 and forces resulting from lift increases as an effect of the kinematic coupling of the hub fluctuation and changes angel of blade pitch horn - F4. If the sum of destabilizing moments will be greater than stabilizing, then the eccentric position of balance will appear.

Destabilization of the rotor hub may occur due to excessive weight of the hub or not enough weight of rotor blades. Because of that autorotation in R22 can be challenging manoeuvre. The length of the triangle base of the hub i.e. spacing of horizontal joints should be large enough. There are two theories about reason of destabilization - the first is an incorrect mounting of rotor blade pitch change horn on trailing edge side (leading edge is correct side). Second is in case of deviation the axis of shaft rotation, and shaft deflection or displacement at the mounting pads. 


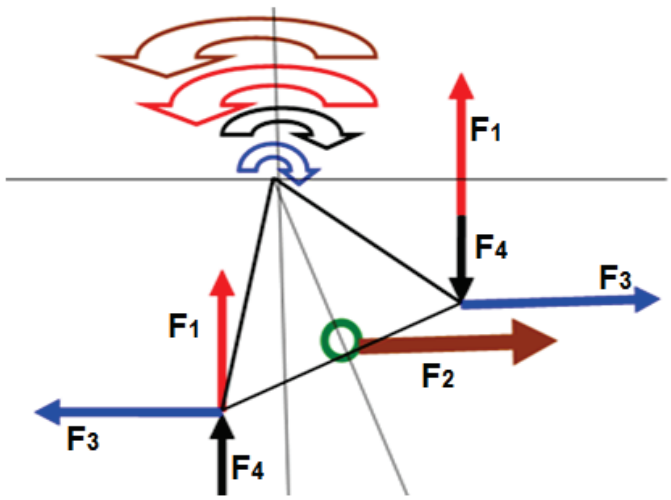

Fig. 3. Simplified model of forces and moments occur on Robinson R22 rotor hub

\section{Conclusion}

Comparison of R22 rotor hub to other two-blade rotors allowed showing the difference that can be cause of high accidents ratio. Analysis of rotor hub stabilization demonstrated the predominance of destabilizing moments which can lead to the occurrence of the eccentric and then to dangerous behaviours of helicopter. Measurements of angles and assessment of rotor behaviour during ground tests (conducted by author) confirmed that helicopter blades perform independent bending movements, and their behaviour should be subject to further analysis. Analysing numerous reports of accidents and statistics, these concluded that the Robinson R22 is a difficult helicopter and requires experience, practice, additional skills and understanding how to proceed during an emergency situation.

No evidence was found to confirm that mechanical factors determine high accident ratio. Due to the high sensitivity of the Robinson R22 control system, it is suggested that during the flight in difficult conditions, pilots should focus all attention on control process, because the problem may be time extended of pilot reaction or limited skills. Excluding the problems of R22 main rotor construction and describing the helicopter as more demanding, the responsibility for accidents is attributed to human factors. It is claimed that R22 just has unique operating characteristics and it might be a very safe helicopter to fly with the proper knowledge of its operation.

Other conclusions resulting from the analysis of accidents are the necessity to increase the pilots' awareness and draw attention to dangerous flight conditions. It is claimed that flights under appropriate conditions and strictly defined rules cannot lead to dangerous situations unless the pilot exceeds the accepted limits. Other causes of accidents can be ruled out due to restrictive pilotage principle, i.e. flight only in certain weather conditions, necessity to attend in additional training conducted by trained instructors.

\section{References}

[1] Bereżański, J., Własności dynamiczne i eksploatacyjne śmigłowców ultralekkich na przykładzie śmigłowca Robinson R22, Prace Instytutu Lotnictwa, s. 117-121, Warszawa 1997.

[2] Bell model 206 series Jet Ranger, Maintenance Manual.

[3] NTSB Investigates Loss-of-control Accidents Among Lightweight Helicopters, Flight Safety Fundation, Helicopter Safety, Canada 1997.

[4] Robinson Helicopter Company R22 loss of main rotor control accidents, Special Investigation Report, Washington 1996.

[5] Robinson model R22, Maintenance Manual.

[6] Wallace, L., You Too Can Hover, Robinson R22 teaches the world how to fly, Flying Magazine, p. 58, March 2002.

[7] https://en.wikipedia.org/wiki/Helicopter_rotor.

[8] https://disciplesofflight.com/sfar-73-a-rule-unlike-any-other-in-aviation.

Manuscript received 30 August 2019; approved for printing 17 December 2019 